\title{
Forces Acting on the Seabed around A Pipeline in Unidirectional Ocean Currents
}

\author{
B. Yang ${ }^{1, *}$, D.-S Jeng ${ }^{2}$, F.P. Gao ${ }^{1}$ and Y.X Wu ${ }^{1}$ \\ ${ }^{1}$ Institute of Mechanics, Chinese Academy of Sciences, Beijing 100190, P. R. China \\ ${ }^{2}$ Division of Civil Engineering, School of Engineering, Physics and Mathematics, University of Dundee, Dundee DD1 \\ $4 H N$, Scotland, U.K
}

\begin{abstract}
In this study, the hydrodynamic forces acting on the seabed around the pipeline in unidirectional ocean currents have been investigated numerically. Two types of seabed are considered, i.e., plane and distorted seabed. The influences of gap between pipeline and seabed on the distribution of forces along the seabed are studied in detail. Computational results show that the pressure at the upstream side of the pipeline gradually decreases and the pressure difference between two sides of the pipeline presents a declining trend with the increase of gap between pipeline and seabed. For the pressure distributions between the distorted seabed and the plane seabed, a double-peaks distribution is observed for the case of distorted seabed, but only one peak exists for the plane seabed. With the increase of gap between pipeline and seabed, the value of peak shear stress along the distorted seabed at the upstream side gradually decreases and the one at the downstream side varies slightly. When the gap ratio reaches 0.7 , the peak shear stress at the upstream side of the pipeline already decreases to a normal level for the case of distorted seabed, while the peak shear stress at downstream side is still a large one for the distorted seabed.
\end{abstract}

Key Words: Hydrodynamic forces, Seabed, Pipeline, Ocean currents, Gap ratio, Numerical simulation.

\section{INTRODUCTION}

In ocean engineering, numerous types of structures have been installed on the seabed, such as offshore platform for exploitation of oil and gas, tension leg, piles and submarine pipelines, etc. Among these, the submarine pipeline is an important structure used widely in offshore oil and gas industry, ship industry, marine fisheries and so on. The submarine pipelines are usually placed in the ocean with several situations, and a horizontal installment is one of the most ordinary ways. After the pipeline is placed on the seabed, it is subject to ocean waves, periodic tides and unidirectional ocean currents. However, in the area of deep sea, the influences of ocean waves and tidal currents upon the pipeline can be ignored, and unidirectional ocean currents are the main hydrodynamic loading acting on pipelines and seabed. Under the action of unidirectional ocean currents and the disturbance due to the existence of the pipeline, the hydrodynamic forces acting on the seabed may be different from those without pipelines. In some circumstances, the forces acting on the sandy seabed may result in the occurrence of sand waves or instability of seabed (e.g. liquefaction) which can cause the buried pipeline to be exposed [1], and the pressure acting on the sandy seabed may induce seepage flow within the sandy seabed. According to the results by Chiew [2] and Sumer et al. [3], when the value of hydraulic gradient induced by the pressure exerting on the sandy seabed reaches up to the critical hydraulic gradient, the liquefaction of sandy soil will occur. Under certain conditions, the

*Address correspondence to this author at the Institute of Mechanics, Chinese Academy of Sciences, Beijing 100190, P. R. China;

E-mail: byang@imech.ac.cn suspension of pipelines with small value of embedment into the sandy seabed and the local scour of sandy seabed around the pipeline will also take place $[4,5]$. Therefore, investigating hydrodynamic forces acting on the seabed will help engineers in design of pipeline to protect sandy seabed from being eroded by fluid, and will be also useful to understand the interactions between pipelines, seabed and ocean currents.

The interactions between cylinders, plane boundary and fluids have attracted wide interests from many researchers. The forces acting on the cylinder and the plane boundary are paid much attention. Taneda [6] made a qualitative observation of the flow around a circular cylinder near a plane boundary in a water tank with Reynolds number $R e=170$. Roshoko et al. [7] investigated the lift and drag coefficients of a circular cylinder near a plane boundary with the gap ratio $\left(e_{0} / D\right)$ from 0 to 6 (in which $e_{0}$ is the gap between the circular cylinder and the boundary, $D$ is the diameter of the cylinder) and found that the drag coefficient $\left(C_{d}\right)$ reaches to a minimum value of 0.8 when the cylinder is touching the boundary. Goktun [8] also found a minimum drag at $e_{0} / D=0$. Bearman \& Zdravkovich [9] studied the flow around a circular cylinder near a plane boundary, and the pressure distributions were measured around the cylinder and along the boundary for various gap ratios $\left(e_{0} / D\right)$ at a Reynolds number of $4.5 \times 10^{4}$. Grass et al. [10] discussed the influence of bed proximity on wake vortices of free spanning pipelines. Taniguchi \& Miyakoshi [11] studied the fluctuations of lift and drag of the cylinder close to a plane boundary and reported that the fluctuating fluid forces are strongly reduced at certain value of the gap. Buresti \& Lanciotti [12] measured the mean and fluctuating forces acting on a circular cylinder near 
a plane surface in cross flow and found that the mean lift coefficient decreased rapidly by increasing the gap size, whereas the mean drag coefficient showed non-monotonic trends with $e_{0} / D$. Lei et al. [13] experimentally investigated the forces of a smooth circular cylinder at Reynolds numbers from $1.30 \times 10^{4}$ to $1.45 \times 10^{4}$ and analyzed the effects of bed proximity upon the forces acting on the cylinder. On the other hand, the fluid forces acting on the boundaries without the existence of structures have also been studied by some researchers. For instance, the work about the investigation of wall shear stress was done by Hanratty \& Campbell [14] and Haritonidis [15]. Sumer et al. [16] studied the wall shear stress by two-component hot-film probe. In addition, Cheng et al. [17] investigated the influence of external turbulence on bed shear stress.

In the aforementioned literature, most research focused on the hydrodynamic forces acting on the structures (e.g. the cylinder) or on the plane boundary (e.g. seabed) without the existence of structures, but few attentions have been paid to the fluid forces acting on the surface of the seabed around the pipeline. In fact, the characteristic of the forces (e.g. the shear stress and the pressure) acting on the surface of the seabed plays an important role about the stability of the seabed $[18,19]$.

This study is aimed at investigating the characteristic of the hydrodynamic forces acting on the seabed around a pipeline. By solving the Navier-Stokes equations and the seepage flow equation, the flow field on the seabed and the seepage flow within sandy seabed will be obtained. Based on the proposed numerical model, the distributions of the pressure and the shear stress along the surface of the seabed will be discussed in detail.

\section{NUMERICAL MODEL}

\section{Governing Equation}

In this study, a two-dimensional problem is considered, as depicted in Fig. (1). The incompressible Reynoldsaveraged Navier-Stokes equations are used to describe the movement of fluid on the seabed as follows:

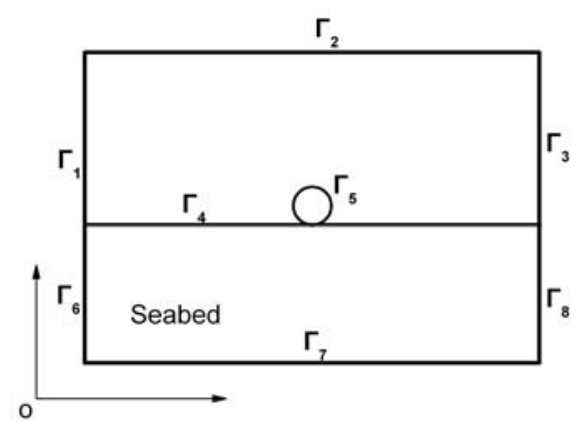

Fig. (1). Sketch of model geometry. $\beta$.

$$
\frac{\partial u_{i}}{\partial x_{i}}=0
$$

$$
\begin{aligned}
\frac{\partial u_{i}}{\partial t}+u_{j} \frac{\partial u_{i}}{\partial x_{j}} & =-\frac{1}{\rho} \frac{\partial p}{\partial x_{i}} \\
& +v \frac{\partial^{2} u_{i}}{\partial x_{j} \partial x_{j}}-\frac{\partial}{\partial x_{j}}\left(\overline{u_{i}^{\prime} u_{j}^{\prime}}\right)
\end{aligned}
$$

where $u_{i}$ is the mean velocity of fluid, $u_{i}^{\prime}$ and $u_{j}^{\prime}$ are the pulse velocity of fluid, $p$ represents the pressure, $\rho$ is the mass density of fluid, $\mu$ denotes the dynamic viscosity coefficient of fluid, $t$ is the time, $x$ is coordinate variable, the subscript $i, j=1,2$ correspond to the directions of $x$ and $y$ respectively.

According to the generalized Boussinesq vortex-viscosity hypothesis [20], the unenclosed term in equation (2) $\overline{-u_{i}^{\prime} u_{j}^{\prime}}$ can be expressed as

$$
\overline{-u_{i}^{\prime} u_{j}^{\prime}}=v_{t}\left(\frac{\partial u_{i}}{\partial x_{j}}+\frac{\partial u_{j}}{\partial x_{i}}\right)-\frac{2}{3} k \delta_{i j}
$$

where $k=\overline{u_{i}^{\prime} u_{i}^{\prime}} / 2$ represents turbulent kinetic energy, $v_{t}$ is the viscosity coefficient of turbulent flow.

The realizable $k-\varepsilon$ model is used to compute the turbulent quantities which can satisfy certain mathematical constraints on the normal stresses. It is consistent with the physics of turbulent flows, compared with other $k-\varepsilon$ models. Moreover, a new eddy-viscosity formula about a variable $\left(C_{\mu}\right)$ is adopted, and a new model equation for dissipation $(\varepsilon)$ based on the dynamic equation of the mean-square vorticity fluctuation is used in realizable $k-\varepsilon$ model.

The transport equations for $k$ and $\varepsilon$ in the realizable $k-\varepsilon$ model are

$$
\begin{gathered}
\frac{\partial k}{\partial t}+\frac{\partial}{\partial x_{i}}\left(u_{i} k\right)=\frac{\partial}{\partial x_{j}}\left[\left(v+\frac{v_{t}}{\sigma_{k}}\right) \frac{\partial k}{\partial x_{j}}\right] \\
+G_{k}-\varepsilon \\
\frac{\partial \varepsilon}{\partial t}+\frac{\partial}{\partial x_{i}}\left(u_{i} \varepsilon\right)=\frac{\partial}{\partial x_{j}}\left[\left(v+\frac{v_{t}}{\sigma_{\varepsilon}}\right) \frac{\partial \varepsilon}{\partial x_{j}}\right] \\
+C_{1} S \varepsilon-C_{2} \frac{\varepsilon^{2}}{k+\sqrt{v \varepsilon}}
\end{gathered}
$$

where $G_{k}$ is related to the production of turbulent kinetic energy and

$$
\begin{aligned}
& G_{k}=-\overline{u_{i}^{\prime} u_{j}^{\prime}} \frac{\partial u_{i}}{\partial x_{j}} \\
& v_{t}=C_{\mu} \frac{k^{2}}{\varepsilon}
\end{aligned}
$$

$C_{\mu}=\frac{1}{A_{0}+A_{s} \frac{k B^{*}}{\varepsilon}}$ 


$$
\begin{aligned}
& B^{*}=\sqrt{S_{i j} S_{i j}+\tilde{\Omega}_{i j} \tilde{\Omega}_{i j}} \\
& \tilde{\Omega}_{i j}=\Omega_{i j}-2 e_{i j l} \omega_{l}
\end{aligned}
$$

where $\Omega_{i j}$ is the mean rotation rate tensor and

$\Omega_{i j}=1 / 2\left(\partial u_{i} / \partial x_{j}-\partial u_{j} / \partial x_{i}\right), e_{i j l}$ is the replacement tensor, $\omega_{l}$ is the fluctuating vorticity, the $\operatorname{term}\left(e_{i j l} \omega_{l}\right)$ is set as zero in this study; The model constants $A_{0}$ and $A_{s}$ are given by

$$
\begin{aligned}
& A_{0}=4.04, A_{s}=\sqrt{6} \cos \varphi \\
& \varphi=\frac{1}{3} \cos ^{-1}(\sqrt{6} W), W=\frac{S_{i j} S_{j l} S_{l i}}{\tilde{S}^{3}}, \\
& \tilde{S}=\sqrt{S_{i j} S_{i j}}, S_{i j}=\frac{1}{2}\left(\frac{\partial u_{j}}{\partial x_{i}}+\frac{\partial u_{i}}{\partial x_{j}}\right) .
\end{aligned}
$$

The coefficient $C_{l}$ in Eq.(5) is defined as

$$
\begin{aligned}
& C_{1}=\max \left[0.43, \frac{\eta}{\eta+5}\right], \\
& \eta=S \frac{k}{\varepsilon}, S=\sqrt{2 S_{i j} S_{i j}}
\end{aligned}
$$

The values of model constants are specified as $C_{1 \varepsilon}=1.44, C_{2}=1.9, \sigma_{k}=1.0$ and $\sigma_{\varepsilon}=1.2$.

For porous seabed, the seepage flow exists within a sandy seabed. In this study, we consider the sandy soil to be homogeneous and isotropic, and the pore water is incompressible. For the case of two-dimensional plane-strain problem, the mathematic equation describing seepage flow can be written as:

$$
\frac{\partial^{2} h}{\partial x^{2}}+\frac{\partial^{2} h}{\partial y^{2}}=0
$$

in which $h(=p / \rho g)$ denotes the pressure water head and $g$ is the gravitational acceleration.

\section{Boundary Conditions}

The typical sketch of the model geometry used in this study is depicted in Fig. (1). $\Gamma_{1}$ is the inflow boundary, where the Dirichlet-type boundary condition, i.e. $u_{1}=u_{0}, u_{2}=0,\left(u_{i}^{\prime} u_{i}^{\prime}\right)^{1 / 2} /\left(u_{i} u_{i}\right)^{1 / 2}=1.2 \%$, and the turbulent viscosity ratio is prescribed, i.e. $v_{t} / v=5$. As shown in the figure, $\Gamma_{3}$ is the outflow boundary, where the diffusion flux in the direction normal to the exit is set to zero for all variables. $\Gamma_{2}$ is defined as the symmetry boundary, namely, zero normal velocity and zero normal gradients of all variables are applied at this boundary. For the wall boundary $\Gamma_{5}$, which is the surface of the pipeline, the logarithmic law of the wall function is adopted, i.e.

$$
\begin{array}{ll}
U^{*}=\frac{1}{\kappa} \ln \left(E y^{*}\right) & \left(\mathrm{y}^{*}>11.2\right) \\
U^{*}=y^{*} & \left(\mathrm{y}^{*}<=11.2\right)
\end{array}
$$

in which

$U^{*}=\frac{u_{P} C_{\mu}^{1 / 4} k_{P}^{1 / 2}}{\tau_{w} / \rho}$ and $y^{*}=\frac{\rho C_{\mu}^{1 / 4} k_{P}^{1 / 2} y_{P}}{\mu}$

where $\kappa$ is von Karman constant and $\kappa=0.42, E$ is the empirical constant and $E=9.79, u_{P}$ is the mean velocity of the fluid at point $\mathrm{P}, k_{P}$ is the turbulence kinetic energy at point $\mathrm{P}$, $y_{P}$ is the distance from point $\mathrm{P}$ to the wall boundary. The boundary condition for $k$ imposed at the wall boundary $\left(\Gamma_{5}\right)$ is

$\frac{\partial k}{\partial n}=0$

in which $n$ is the local coordinate normal to the solid boundary. The production of kinetic energy $\left(G_{k}\right)$ and its dissipation rate $(\varepsilon)$ at the wall boundary-adjacent cells are computed on the basis of the local equilibrium hypothesis. Under this assumption, the production of $k$ and its dissipation rate are assumed to be equal in the wall boundary-adjacent control volume. The production of $k$ is computed from

$G_{k}=\tau_{w} \frac{\tau_{w}}{\kappa \rho C_{\mu}^{1 / 4} k_{P}^{1 / 2} y_{P}}$

The $\varepsilon$ is computed from

$\varepsilon_{P}=\frac{C_{\mu}^{3 / 4} k_{P}^{3 / 2}}{\kappa y_{P}}$

When the N-S equations, i.e. Eqs. (1) and (2), are solved, the surface of the seabed $\left(\Gamma_{4}\right)$ is specified as the same type as the boundary $\Gamma_{5}$, i.e. the wall boundary. However, when the seepage flow equation (see Eq.(13)) is solved, $\Gamma_{4}$ is a pressure water head boundary, where $h=h_{1}(x)$ is applied. $\Gamma_{6}$, $\Gamma_{7}$ and $\Gamma_{8}$ are set as impermeable boundary, i.e. $\partial h / \partial n=0$.

\section{Numerical Method}

The finite volume method is used to discretize the N-S equation. The SIMPLE algorithm [21] is adopted to deal with pressure-velocity coupling. The quadrilateral and triangular elements are used in flow model. To improve the precision of computation, the grids around the pipeline are refined. The size of grid is specified on the basis of the study of mesh dependence. The seepage flow equation is solved by finite element method with Lagrange quadratic triangular elements. The N-S equation and seepage flow equation are solved respectively. At first, the N-S equation is solved, and the pressure along the surface of sandy seabed is obtained. Then the seepage flow equation is solved by defining the pressure along the surface of seabed from the solution of $\mathrm{N}-\mathrm{S}$ equation at the boundary $\Gamma_{4}$. 


\section{Verification of Numerical Model}

To verify the validation of the numerical model, the comparison is made upon the mean lift and drag forces acting on the pipe near the rigid boundary and the pressure along the boundary between the numerical and experimental results in this section. The experimental results reported by Buresti et al. [12] and Lei et al. [13] are used to verify the present model, as depicted in Fig. (2).

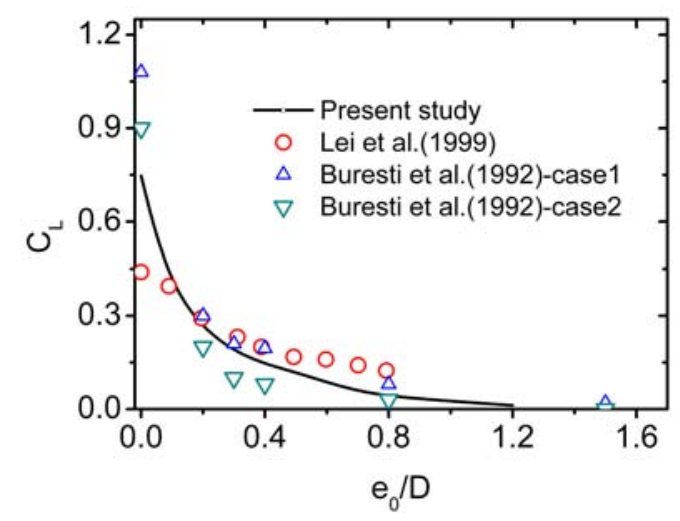

Fig. (2a). The comparison upon the mean lift forces acting on the pipe between numerical and experimental results. (In the work by Buresti et al. [12]: $R e=1.89 \times 10^{5}, 1.42 \times 10^{5}$, in the work by Lei et al. [13]: $\left.R e=1.36 \times 10^{4}\right)$.

The lift force coefficient $\left(C_{L}\right)$ and drag force coefficient $\left(C_{D}\right)$ are defined respectively as:

$$
\begin{aligned}
& C_{L}=\frac{F_{L}}{1 / 2 \rho u_{\infty}^{2} D L} \\
& C_{D}=\frac{F_{D}}{1 / 2 \rho u_{\infty}^{2} D L}
\end{aligned}
$$

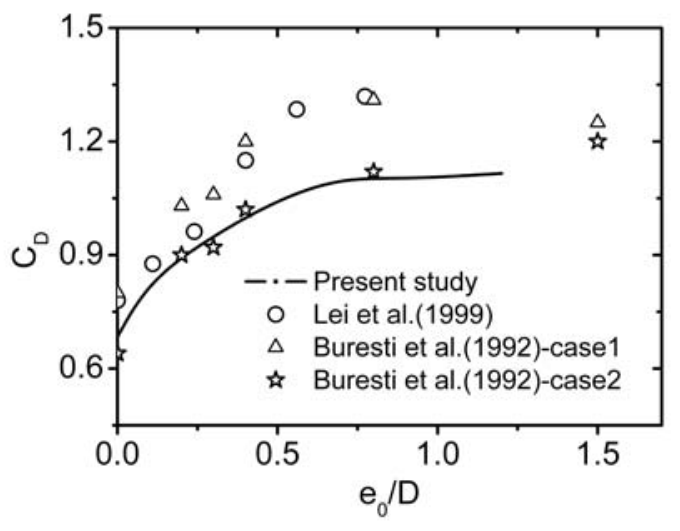

Fig. (2b). The comparison upon the mean drag forces acting on the pipe between numerical results and experimental results.(Re $=1.89 \times 10^{5}, 1.42 \times 10^{5}$ in work by Buresti et al. [12], $R e=1.36 \times 10^{4}$ for the work by Lei et al. [13]).

where $F_{L}$ is the lift force acting on the pipe, $F_{D}$ is the drag one, $u_{\infty}$ is the inflow velocity, $\mathrm{L}$ is the length of the pipe. It is noted that the Reynolds number in their experiments mentioned above are within the subcritical regime. The differ- ences between Cases 1 and 2 by Buresti et al. [12] are the different thickness of boundary layer. The thickness of case 1 is $0.1 D$ at the cylinder axis position, while it is $1 D$ for the case 2 . The thickness of boundary layer is $0.14 D$ for the results by Lei et al. [13] and $0.2 \mathrm{D}$ for this study.

It can be seen from Fig. (2) that the mean lift coefficient $\left(C_{L}\right)$ computed by the present model is about 0.75 at $e_{0} / D=0$ and 0.15 at $e_{0} / D=0.4$. When the gap-todiameter ratio $\left(e_{0} / D\right)$ increases to the value of 1.2 , the mean lift coefficient almost tends to be zero. The computational mean lift coefficients agree with those of experiments well. The mean drag coefficient $\left(C_{D}\right)$ presented in this study is about 0.68 at $e_{0} / D=0$ and 0.99 at $e_{0} / D=0.4$. The drag coefficient increases to 1.09 for the case of $e_{0} / D=0.8$, which is a little lower than experimental results. However, the trend of drag force versus gap ratio $\left(e_{0} / D\right)$ is consistent.

Fig. (3) presents the comparison of the mean (timeaveraged) pressure distribution along the plane boundary. The pressure coefficient $\left(C_{p}\right)$ is defined as:

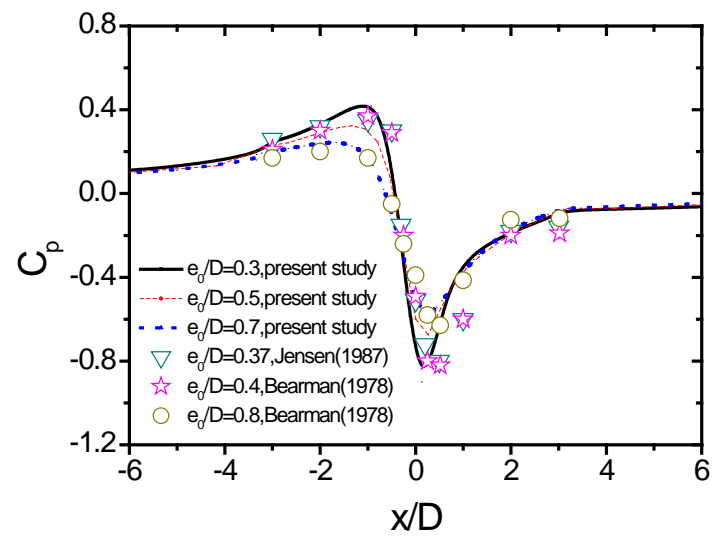

Fig. (3). The comparison upon the mean pressure along the plane boundary between present model and experimental results (the experimental parameters in the work by Jensen [22] are: $\mathrm{e}_{0} / \mathrm{D}=0.37$, $\mathrm{Re}=0.7 \times 10^{4} ; R e=4.8 \times 10^{4}$ for the work by Bearman \& Zdravkorich [9]).

$$
C_{p}=\frac{p-p_{0}}{1 / 2 \rho u_{\infty}^{2}},
$$

where $p$ is the static pressure, $p_{0}$ is the reference pressure, which is set as $101325 \mathrm{~Pa}$. It is indicated from the figure that the variation of mean pressure coefficient along the plane boundary agrees well with the experimental results by Bearman [9] and Jensen [22].

\section{RESULTS AND DISCUSSIONS}

In this study, the model parameters are set as follows: the height of flow region is $30 \mathrm{D}$, and the length is $60 \mathrm{D}$. The distance between the center of the pipeline and the inflow boundary is $30 \mathrm{D}$. Therefore, it is $30 \mathrm{D}$ from pipeline center to outflow exit. The depth of seabed is 30D. The mass density of fluid is set as $998 \mathrm{~kg} / \mathrm{m}^{3}$, and the dynamic viscosity coefficient of the fluid is $0.001 \mathrm{~Pa} \cdot s$. The diameter of the pipeline is set as $0.6 \mathrm{~m}$ and the inflow velocity is specified as $0.2 \mathrm{~m} / \mathrm{s}$. Therefore, the Reynolds number is about $1.19 \times 10^{5}$ 
in this study. Two types of seabed are considered, i.e. the plane seabed and the distorted seabed, as depicted in Fig. (4). For the distorted seabed, the main emphasis is laid on the various arc-shaped deformation of seabed beneath the pipeline.
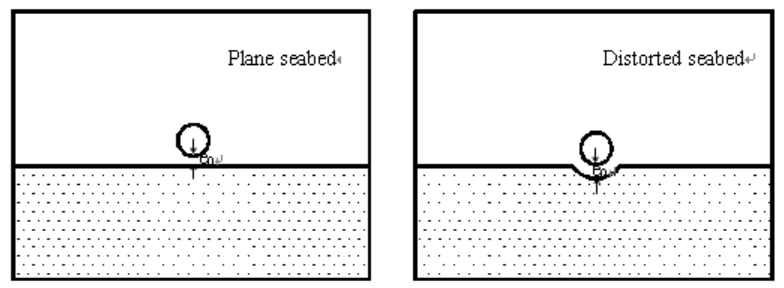

Fig. (4). The sketch of two types of seabed.

\section{Characteristic of Flow Around the Pipeline and Seepage Flow within Sandy Bed}

An example for the plane sandy seabed with a pipeline is taken in this section. Fig. (5) illustrates the typical distribution of flow velocity on the seabed with a pipeline and that of hydraulic gradient induced by the flow on the seabed at some time $\mathrm{t}_{0}$. It is noted that the gap between the pipeline and the seabed is zero in Fig. (5). There exists an apparent wake flow region within which the magnitude of velocity is smaller than that outside the wake region, and the maximum velocity appears near the top surface of the pipeline, as can be seen in Fig. (5). The value of hydraulic gradient close to the bottom of the pipeline is much larger than that far from the pipeline, which also indicates that the maximum hydraulic gradient occurs at the region near the pipeline.

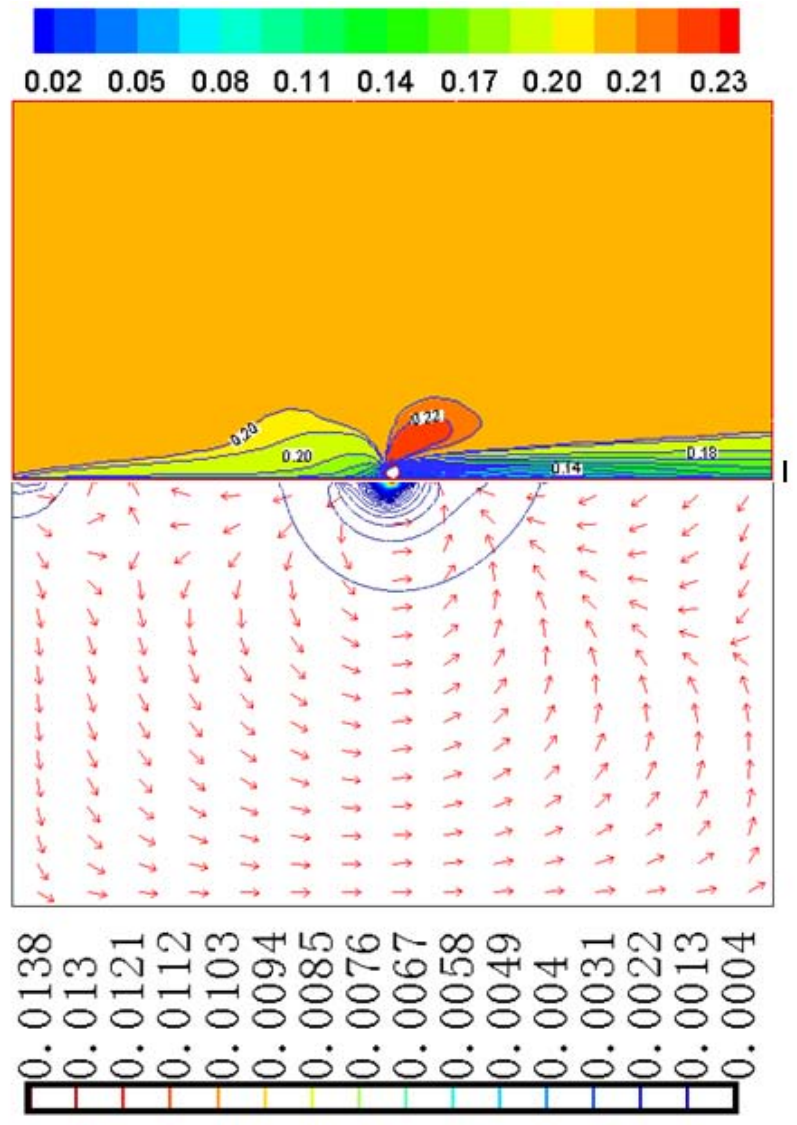

Fig. (5). Distribution of velocities on the sandy seabed and seepage flow within a sandy seabed.

\section{Pressure and Shear Stress Along the Seabed}

The distribution of mean (time-averaged) pressure along the plane seabed with various gap-to-diameter ratios is illustrated in Fig. (6). It can be seen from the figure that there is a jump of pressure between the upstream and downstream sides of the pipeline. With the increase of gap between pipeline and seabed the amplitude of the pressure at the upstream side of the pipeline gradually decreases. The pressure difference between two sides of the pipeline presents a declining trend with the increasing gap.

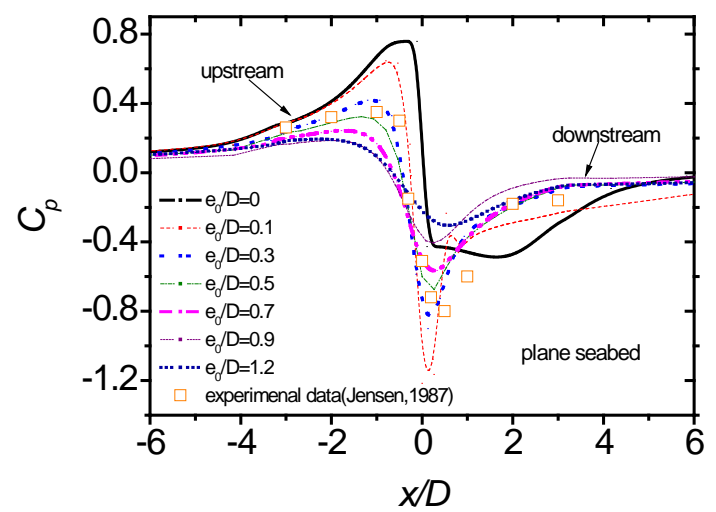

Fig. (6). The distribution of mean pressure along the surface of plane seabed.

Fig. (7) gives the mean (time-averaged) pressure distribution along the distorted surface of seabed (arc-shaped). It is noted that the results for the case of $e_{0} / D=0$ is also included in the figure for reference. It can be seen from the figure that there are some differences for the pressure distribution between the distorted seabed and the plane seabed. Two pressure peaks are observed for the case of distorted seabed, but only one exists for plane seabed. Within the distorted gap the pressure along the surface undergoes an ascending and descending process.

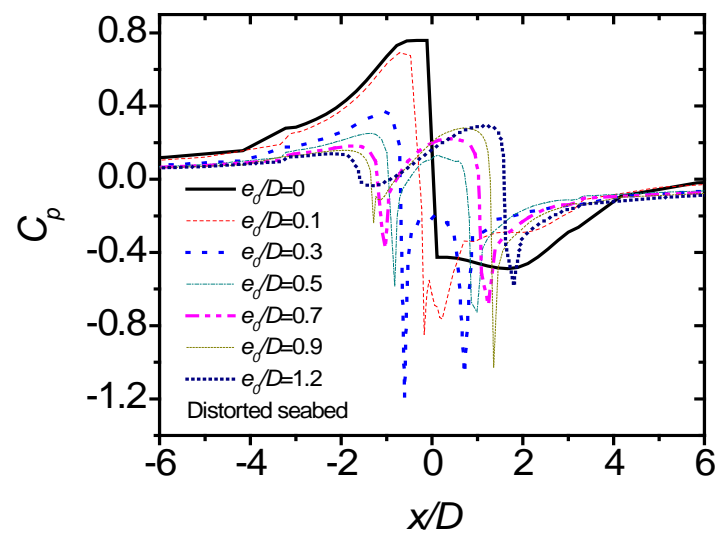

Fig. (7). The distribution of mean pressure along the surface of distorted seabed.

The distribution of mean (time-averaged) shear stress along the surface of plane seabed with seven gap ratios, i.e. $e_{0} / D=0,0.1,0.3,0.5,0.7,0.9,1.2$, are shown in Fig. (8), where the friction factor $\left(C_{f}\right)$ in the figure is defined as

$$
C_{f}=\frac{\tau_{b}}{1 / 2 \rho u_{\infty}^{2}},
$$


in which $\tau_{b}$ is the bed shear stress. It can be observed from the figure that when the pipeline contacts the surface of seabed, the shear stress within the range of $x / D<0.5$ along the surface of seabed is very small. And at the place of $x=1.5 D$ there exists a peak of shear stress. For the case of positive value of gap ratio (e.g. $e_{0} / D=0.1,0.3 \ldots$ ), there is also a peak of shear stress, but it occurs beneath the bottom of the pipeline.

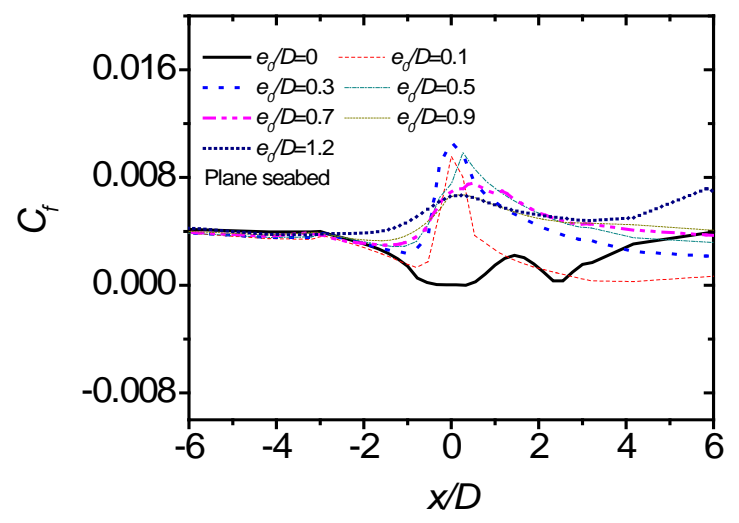

Fig. (8). The distribution of mean shear stress along the surface of plane seabed.

Fig. (9) presents the distribution of mean (time-averaged) shear stress along the distorted seabed with several gap ratios. It is shown in the figure that there are two peaks of shear stress for the case of distorted seabed, which occur at the upstream and downstream sides of the pipeline. With the increase of gap between pipeline and seabed, the value of peak shear stress at the upstream side gradually decreases and that at the downstream side varies little. When the gap ratio reaches up to 0.7 , the peak shear stress at the upstream side of the pipeline already decreases to a normal level, while the peak shear stress at downstream side is still a large one. It means that the place at downstream side of the pipeline will be scoured much intensively compared with that at upstream side. When the gap between pipeline and seabed is small (e.g. $e_{0} / D=0.1$ ), the shear stress along the gap presents a big value. With the increasing gap ratio the place of maximum shear stress gradually moves to the downstream side of the pipeline.

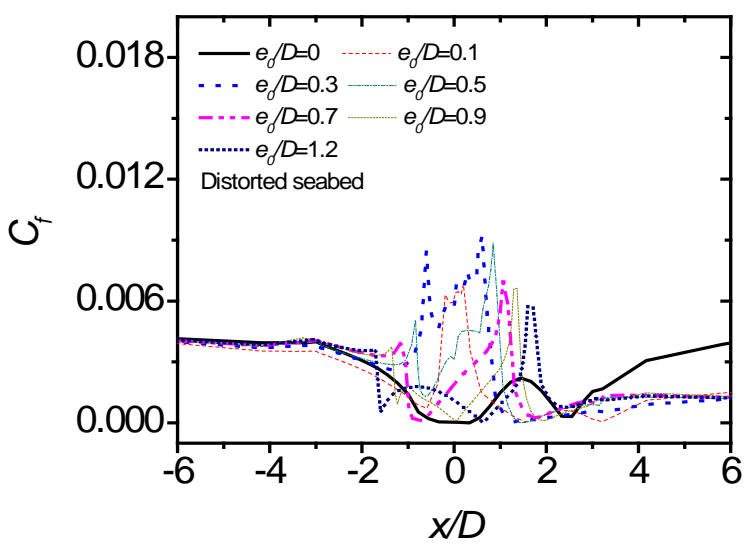

Fig. (9). The distribution of mean shear stress along the surface of distorted seabed.
A comparison of pressure distribution along plane and distorted seabed was made, as shown in Fig. (10). It is observed from Fig. (10) that the pressure difference between two sides of pipeline for the case of distorted seabed is lower than that for plane seabed. Fig. (11) gives the distribution of shear stress along two types of seabed for $e_{0} / D=0.1$ and 0.5 . It can be seen from the figure that the shear stress along the gap between pipeline and seabed for the distorted seabed is smaller than that for the plane seabed. This concludes that under the condition of same gap ratio the distorted sandy seabed is more stable than the plane sandy seabed.

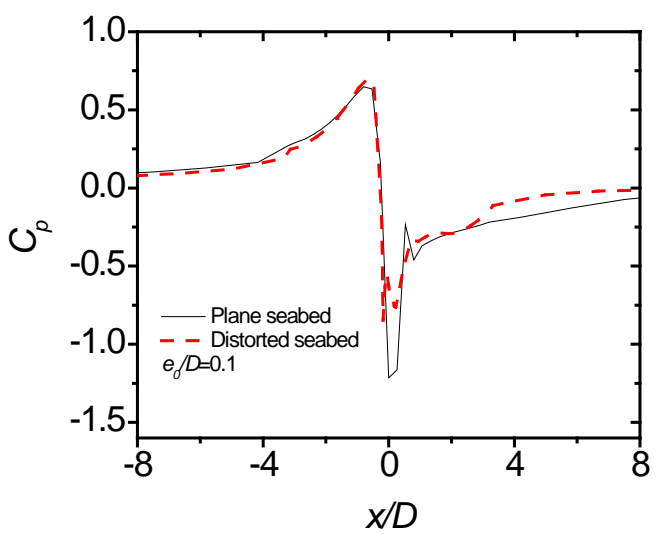

Fig. (10a). The comparison of mean pressure distribution along the surface between plane and distorted seabed- $e_{0} / D=0.1$.

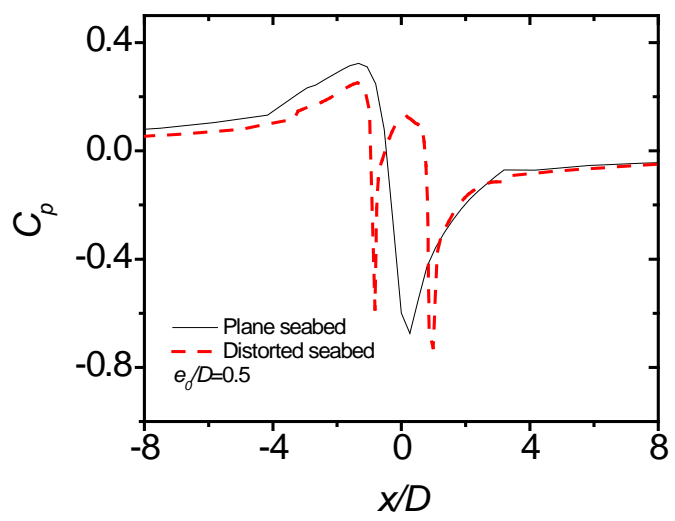

Fig. (10b). The comparison of mean pressure distribution along the surface between plane and distorted seabed- $e_{0} / D=0.5$.

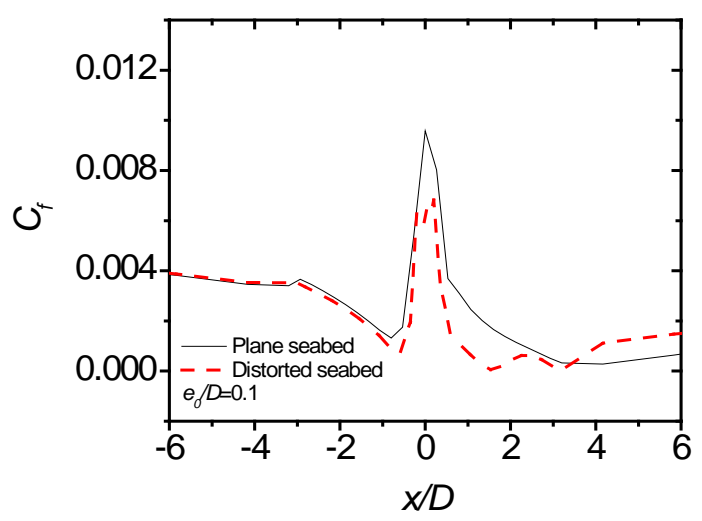

Fig. (11a). The comparison of mean shear stress along the surface between plane and distorted seabed- $e_{0} / D=0.1$. 


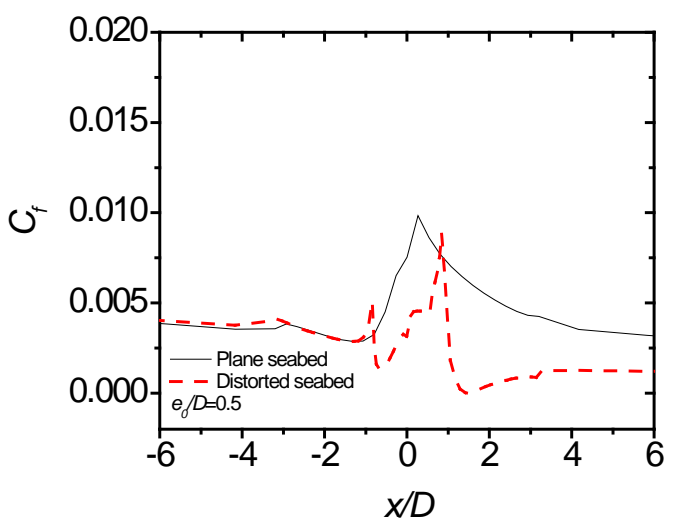

Fig. (11b). The comparison of mean shear stress along the surface between plane and distorted seabed- $e_{0} / D=0.5$.

Fig. (12) plots three types of distorted surface of seabed. The gaps between pipeline and seabed are $0.1 D, 0.5 D$ and $0.48 D$. It is noted that the distorted boundaries for the first two cases are arc-shaped and designed manually. The distorted boundary for the third case is an experimental profile which corresponds to the equilibrium scour profile of the sandy bed under action of certain unidirectional flow. In the experiments, at the initial time the pipeline just contacts to the surface of sandy bed, i.e. the gap is zero, then the scour occurs until the equilibrium stage. In the equilibrium stage, the profile of surface of sandy bed changes little and for the clear-water scouring case the shear stress along the surface induced by fluid drops below the value of critical shear stress at which the sand grain will begin to move. The details about the experimental profile can be referenced to the work by Yang et al. [23].
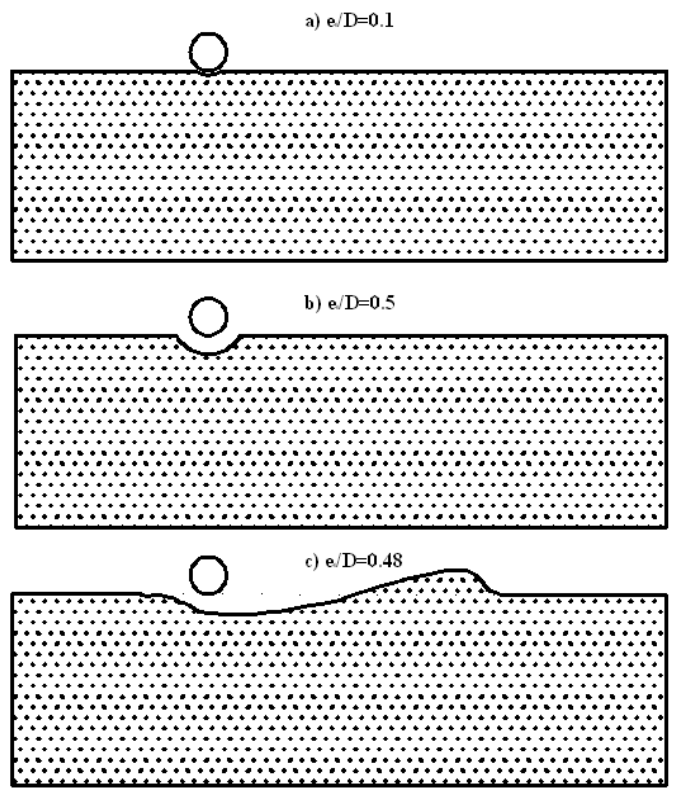

Fig. (12). The schematic of various distorted surface of seabed.

Fig. (13) presents the distribution of shear stress along the three types of distorted surface of seabed. It can be seen from the figure that the shear stress along the surface of sandy bed obtained from experiments is smaller than that from the arc-shaped surface. It means that the arc-shaped surface of sandy bed corresponding to the first two cases in
Fig. (12) will be eroded more easily than the surface corresponding to the third case in Fig. (12) under the action of same steady flow.

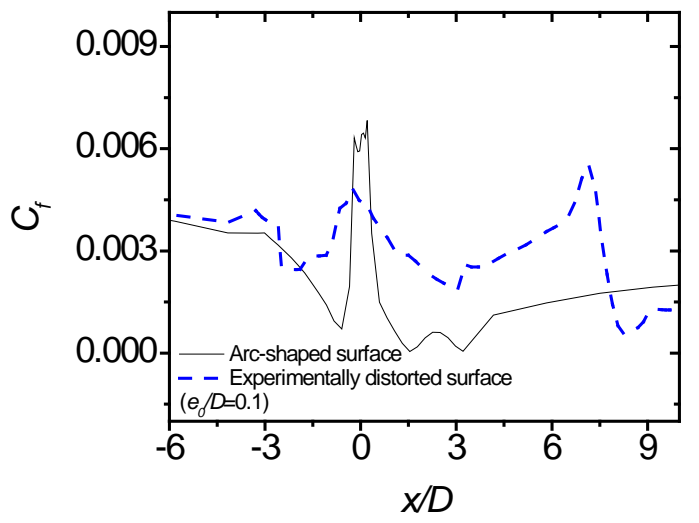

Fig. (13a). The comparison of mean shear stress distribution along the distorted surface- $e_{0} / D=0.1$ versus experimental profile.

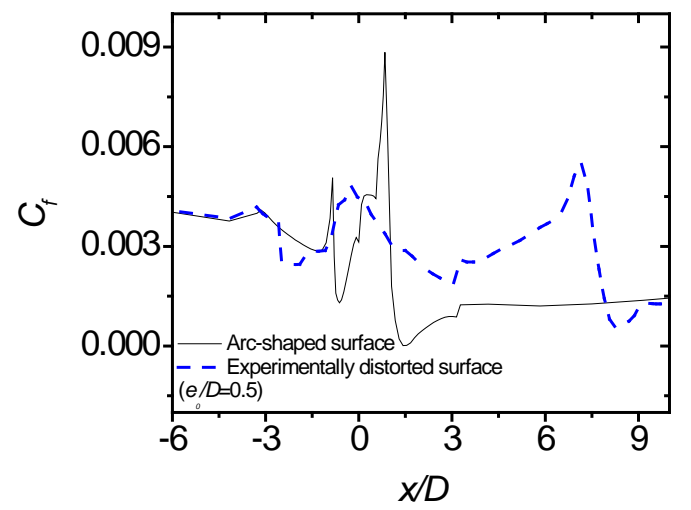

Fig. (13b). The comparison of mean shear stress distribution along the distorted surface- $e_{0} / D=0.5$ versus experimental profile.

\section{CONCLUDING REMARKS}

In this study, we investigated the hydrodynamic forces acting on the two types of sandy seabed (i.e. plane seabed and distorted seabed) with a pipeline. The characteristics of flow field on the seabed around the pipeline and seepage flow within the sandy seabed under the pipeline are analyzed. The pressure and shear stress distributions along the seabed for the case of several gaps between seabed and pipeline are discussed in detail.

With the increase of gap between pipeline and seabed the pressure at the upstream side of the pipeline gradually decreases and the pressure difference between two sides of the pipeline presents a declining trend. For the pressure distribution between the distorted seabed and the plane seabed, there are two pressure peaks for distorted seabed, but only one exists for plane seabed.

For the case of positive value of gap ratio (e.g. $e_{0} / D=$ $0.1,0.3 \ldots)$, there is one peak of shear stress beneath the bottom of the pipeline along the surface of plane seabed, while there are two peaks of shear stress for the case of distorted seabed, which occur at the upstream and downstream sides of the pipeline.

With the increase of gap between pipeline and seabed, the value of peak shear stress along the distorted seabed at 
the upstream side gradually decreases and the one at the downstream side varies slightly. When the gap ratio reaches up to 0.7 the peak shear stress at the upstream side of the pipeline already decreases to a normal level, while the peak shear stress at downstream side is still a large one for the distorted seabed. Under the condition of same gap ratio the distorted sandy seabed is more stable than the plane sandy seabed.

\section{ACKNOWLEDGEMENTS}

This work is financially supported by National Natural Science Foundation of China (Grant No. 50509022 and No. 10532070), and the Eleventh Five-Year Plan of Chinese Academy of Sciences (Grant No. KJCX2-YW-L02).

\section{REFERENCES}

[1] R. J. S. Whitehouse, J. S. Damgaard, and D. N. Langhorne, "Sand waves and seabed engineering: application to submarine cables", Proceedings of the Marine Sandwave Dynamics, Lille, France, 2000, pp. 227-234.

[2] Y.M. Chiew, "Mechanics of local scour around submarine pipelines", J. Hydraul. Eng., ASCE, vol. 116(4), pp. 515-529, 1990.

[3] B.M. Sumer, C. Truelsen, and J. Fredsøe, "Onset of scour below pipelines and self-burial", Coast. Eng., vol. 42, pp. 313-335, 2000.

[4] B.M. Sumer, H.R. Jensen, Y. Mao, and J. Fredsøe, "Effect of leewake on scour below pipelines in current", J. Waterways Port, Coastal \& Ocean Engineering, ASCE, vol. 114(5), pp. 599-614, 1988.

[5] L. Cheng, and F. Li, "Modelling of local scour below a sagging pipeline", Coast. Eng. J., vol. 45(2), pp.189-210, 2003.

[6] S. Taneda, "Experimental investigation of vortex streets", J. Phys. Soc. Japan., vol. 20, pp. 1714-1721, 1965.

[7] A. Roshko, A. Steinolfson, and V. Chattoorgoon, "Flow forces on a cylinder near a wall or near another cylinder", Proceeding. $2^{\text {nd }}$ US Conference. Wind Engineering Research, Fort Collins 1975, 4-15.

[8] S. Goktun, "The drag and lift characteristics of a cylinder placed near a plane surface," M.Sc. thesis, Naval Postgraduate school, Monterey, California, 1975.

[9] P. W. Bearman, and M. M. Zdravkovich, "Flow around a circular cylinder near a plane boundary", J. Fluid Mech., vol. 109, pp. 3348, 1978.
[10] A. J. Grass, J. A. Bray, P. W. J. Raven, and R. J. Stuart, “The influence of boundary layer velocity gradients and bed proximity on vortex shedding from free spanning pipelines", J. Energ. Resour., ASME, vol. 106, pp. 70-78, 1984.

[11] S. Taniguchi, and K. Miyakoshi, "Fluctuating fluid forces acting on a circular cylinder and interference with a plane wall", Exp. Fluids, vol. 9, pp. 197-204, 1990.

[12] G. Buresti, and A. Lanciotti, "Mean and fluctuating forces on a circular cylinder in cross-flow near a plane surface", J. Wind Eng. Ind. Aerod., vol. 41-44, pp. 639-650, 1992.

[13] C. Lei, L. Cheng, and K. Kavanagh, " Re-examination of the effect of a plane boundary on force and vortex shedding of a circular cylinder", J. Wind Eng. Ind. Aerod., vol. 80, pp. 263-286, 1999.

[14] T. J. Hanratty, and J. A. Campbell, "Measurement of wall shear stress", in Fluid Mechanics Measurements, R. J. Goldstein Ed. Hemisphere, Washington, DC, pp. 559-615, 1983.

[15] J. H. Haritonidis, "The measurement of wall shear stress", in Advances in Fluid Mechanics Measurements, M. Gad-el-Hak Ed. Lecture Notes Eng., vol. 45, pp. 229-261,1989.

[16] B. M. Sumer, M. M. Arnskov, N. Christiansen, and F. E. Jorgensen, "Two-component hotfilm probe for measurements of wall shear stress", Exp. Fluids, vol. 15(6), pp. 380-384, 1993.

[17] N. S. Cheng, B. M. Sumer, and J. Fredsøe, "Investigation of bed shear stresses subject to external turbulence", In. J. Heat Fluid Fl., vol. 24(6), pp. 816-824, 2003.

[18] B. M. Sumer, and J. Fredsøe, "The mechanics of scour in the marine environment", Singapore, world scientific, 2002.

[19] F. P. Gao, S. M. Yan, B. Yang, and Y. X. Wu, "Ocean currentsinduced pipeline lateral stability", J. Eng. Mech., ASCE, vol. 133(10), pp. 1086-1092, 2007.

[20] Boussinesq, J., "Théorie de l'Écoulement Tour billa nt", Mem. Présentés par Divers Savants Acad. Sci. Inst. Fr., vol. 23, pp. 4650, 1877.

[21] S.V. Patankar, and D.B. Spalding, "A calculation procedure for heat, mass and momentum transfer in threedimensional parabolic flows." Int. J. Heat Mass Tran., vol. 15, pp. 1787-1806, 1972.

[22] B.L. Jensen, "Large-scale vortices in the wake of a cylinder placed near a wall." Proceeding $2^{\text {nd }}$ international conference on laser anemometry-advances and applications, Strathclyde, UK. pp. 153-163, 1987.

[23] B. Yang, F.P. Gao, and Y.X. Wu, "Experimental study on local scour of sandy seabed under submarine pipeline in unidirectional currents." Eng. Mech., vol. 25(3), pp. 206-210, 2008. 